\title{
Desgaste y corrosión de recubrimientos de PLGA sobre titanio en contacto con hueso y fluido sinovial
}

\author{
Wear and corrosion of PLGA coatings on \\ titanium in contact with bone and \\ synovial fluid
}

\author{
Anderson Sandoval Amador ${ }^{1,2}$, Laura Andrea Ramírez Prada ${ }^{1}$, \\ Sergio Andrés Infante Villalba ${ }^{1}$, Hugo Armando Estupiñán Duran ${ }^{3}$, \\ Darío Yesid Peña Ballesteros ${ }^{1,4}$
}

\footnotetext{
${ }^{1}$ Grupo de Investigaciones en Corrosión - GIC - Universidad Industrial de Santander - UIS, Bucaramanga, Soto, Colombia.

${ }^{2}$ Grupo de Investigación en Diseño de Materiales - DIMAT - Unidades Tecnológicas de Santander - UTS, Soto, Bucaramanga, Colombia.

${ }^{3}$ Grupo de Ciencia y Tecnología de Materiales - Universidad Nacional de Colombia, Medellín, Santa Fé, Soto, Colombia.

${ }^{4}$ Centro de Materiales y Nanociencias, Universidad Industrial de Santander - UIS, Bucaramanga, Soto, Colombia.

e-mail: dypena@uis.edu.co, anderson84f@gmail.com, haestupiñand@unal.edu.co
}

\section{RESUMEN}

En este trabajo se evaluó el sinergismo entre el desgaste y la corrosión, en probetas de titanio recubiertas por PLGA. Los ensayos se realizaron en una máquina de tribocorrosión, teniendo en cuenta dos variables: velocidad de rayado y carga aplicada. Se adaptó un bipotenciostato para obtener las curvas potenciostáticas y Tafel en condiciones de reposo, en movimiento y desgaste-corrosión. La topografía de superficies se observó mediante un microscopio Imager Z1 de Carl-Zeiss. Esta información, conjuntamente con los resultados de los ensayos electroquímicos, se usó para establecer los mecanismos sinergísticos y de degradación del material en las diferentes condiciones de ensayo. De los resultados obtenidos en este trabajo, se observó que el sinergismo es el factor más importante de la degradación del material con un porcentaje de $82 \%$ de tal forma que las muestras de titanio con recubrimiento de PLGA presentaron desgaste favorecido por corrosión.

Palabras clave: desgaste, corrosión, tribocorrosión, titanio, PLGA, fluido sinovial.

\section{ABSTRACT}

In this work, the synergism between wear and corrosion was evaluated in titanium specimens covered by PLGA. The tests were carried out in a tribocorrosion machine, taking into account two variables: scratch velocity and applied load. A bipotentiostat was adapted to obtain the potentiostatic curves and Tafel in resting, moving and wear-corrosion conditions. Surface topography was observed using a Carl-Zeiss Imager Z1 microscope. This information, together with the results of the electrochemical tests, was used to establish the synergistic and degradation mechanisms of the material under the different test conditions. From the results obtained in this work, it was observed that the synergism is the most important factor of the degradation of the material with a percentage of $82 \%$ in such a way that the samples of titanium with PLGA coating showed wear favored by corrosion.

Keywords: wear, corrosion, tribocorrosion, titanium, PLGA, synovial fluid.

\section{INTRODUCCIÓN}

El titanio comercialmente puro ( $\mathrm{Ti} \mathrm{cp}$ ) y sus aleaciones son ampliamente usados en aplicaciones biomédicas como materiales de implante debido al excelente conjunto de propiedades como alta relación resistencia/peso, propiedades mecánicas adecuadas, alta resistencia a la corrosión y buena biocompatibilidad [1]. La alta resistencia a la corrosión del titanio y sus aleaciones se debe a que naturalmente forma una capa pasiva de $\mathrm{TiO}_{2}$ la 
cual tiene unos pocos nanómetros de espesor. Por otro lado, la superficie del titanio tiene la capacidad de iniciar las reacciones químicas que permiten la formación de apatitas, aunque la capacidad que tiene para permitir la formación de hueso nuevo es muy pobre [2]. Con el fin de mejorar esta falencia de las superficies de titanio se han venido desarrollando un gran número de estudios, los cuales apuntan a modificar la morfología, la composición química, la energía superficial, la resistencia a la corrosión y al desgaste de las superficies de este material [3-7].

Uno de los métodos de modificación superficial que ha generado muy buenos resultados en cuanto a la adhesión celular, la formación de hueso y la corrosión ha sido la aplicación de recubrimientos poliméricos sobre las superficies de titanio. Algunos de los polímeros que se han aplicado como recubrimientos son ácido poliláctico PLA [8-10], ácido poliglicólico PGA [11], policaprolactona PCl [12], quitosano [13] y colágeno $[14,15]$. Estos recubrimientos al momento de degradarse liberan sustancias que son metabolizadas por las células y de esta manera se promueven las condiciones para lograr la adhesión y proliferación celular. Así mismo, el ácido poli-láctico-co-glicólico PLGA es uno polímero reabsorbible que ha sido empleado para aplicaciones ortopédicas ya que provee una matriz biodegradable que facilita la adhesión, proliferación y diferenciación de células óseas [16-19].

En este orden de ideas, el objetivo de este estudio fue evaluar el desgaste y la corrosión que sufre un recubrimiento de ácido poli-láctico-co-glicólico PLGA al estar en contacto con hueso y fluido sinovial.

\section{MATERIALES Y MÉTODOS}

Inicialmente se fabricó un indentador a partir de hueso animal. Para ello se partió de una muestra comercial de hueso bovino el cual se liofilizó, cortó y secó (Figura 1a), luego las muestras de hueso se maquinaron en forma de indentador como se puede apreciar en la Figura $1 \mathrm{~b}$.
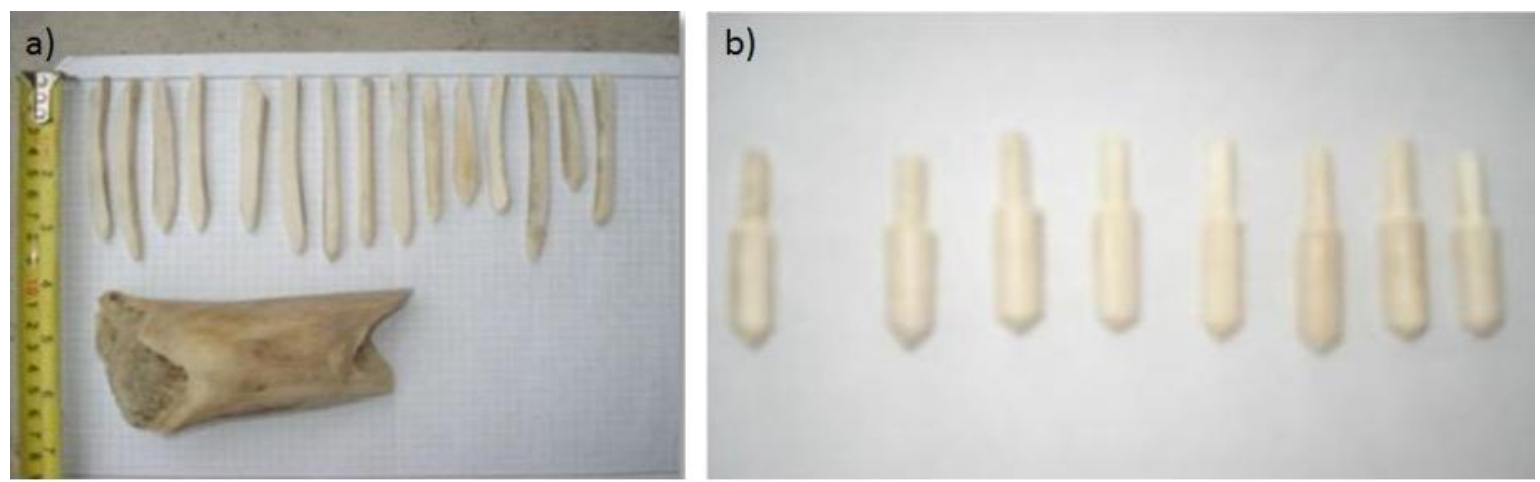

Figura 1: a) Hueso animal liofilizado, b) Indentador de hueso animal.

Se fabricaron probetas de titanio comercialmente puro (Ti cp) en forma de disco con diámetro de 12 $\mathrm{mm}, 3 \mathrm{~mm}$ de espesor, y área expuesta de $4,52 \mathrm{~cm}^{2}$. Se realizó un procedimiento de preparación superficial a cada probeta, el cual consistió en desbaste con papel de carburo de silicio desde 120, 240, 360, 400 hasta 600, enjuague y desengrase en baño ultrasónico con etanol por 10 minutos y secado con aire caliente. Este procedimiento está basado en la norma ASTM G1-90, 1999 [20].

Posteriormente las probetas fueron recubiertas con PLGA por deposición electroforética para lo cual se polarizaron las muestras a $100 \mathrm{mV}$ vs $\mathrm{Ag} / \mathrm{AgCl}$ durante 15 minutos. La síntesis del copolímero PLGA se realizó mediante el método de mezcla/agitación de solvente, utilizando como solvente cloroformo. Los homopolímeros de ácido láctico PLA y ácido glicólico PGA fueron disueltos individualmente en cloroformo durante una hora en una proporción de 70:30 v/v.

Tabla 1: Composición de la solución Ringer.

\begin{tabular}{l|l|l|l|l}
\hline Composición & $\mathbf{N a C l}$ & $\mathrm{KCl}$ & $\mathrm{Na}_{2} \mathrm{HPO}_{4}$ & $\mathrm{KH}_{2} \mathrm{PO}_{4}$ \\
\hline $\mathrm{g} / \mathrm{L}$ & 8,06 & 0,22 & 1,15 & 0,20 \\
\hline
\end{tabular}

Haciendo uso de curvas de polarización Tafel se determinó la velocidad de corrosión para cada una de las muestras, las cuales se evaluaron en contacto con una solución simulada de fluido sinovial (Solución Ringer) con un pH de 7,4 (ver composición en la Tabla 1). La velocidad de barrido empleada para la realización 
de estas pruebas fue de 7,5 $\mathrm{mV} / \mathrm{min}$ en una ventana de potencial de $\pm 650 \mathrm{mV}$ alrededor del potencial de circuito abierto el cual se midió durante 1 minuto, estas pruebas se llevaron a cabo teniendo en cuenta las indicaciones de la norma ASTM G3-89, 2010 [21].

Los ensayos de tribocorrosión se realizaron con un indentador cargado por medio de un sistema de pesos menores a $1 \mathrm{~N}$, sumergido dentro de una celda, lo cual permitió contener la solución Ringer que simula el entorno corrosivo (Figura 2). Las mediciones del sinergismo desgaste-corrosión, se realizaron según la norma ASTM G 119-09, 2009 [22]. Los ensayos potenciostáticos se realizaron basados en la norma ASTM G5-94, 2011 [23].

La morfología de las superficies de titanio y titanio recubierto con PLGA se analizó usando un Microscopio Imager Z1 de Carl-zeiss.

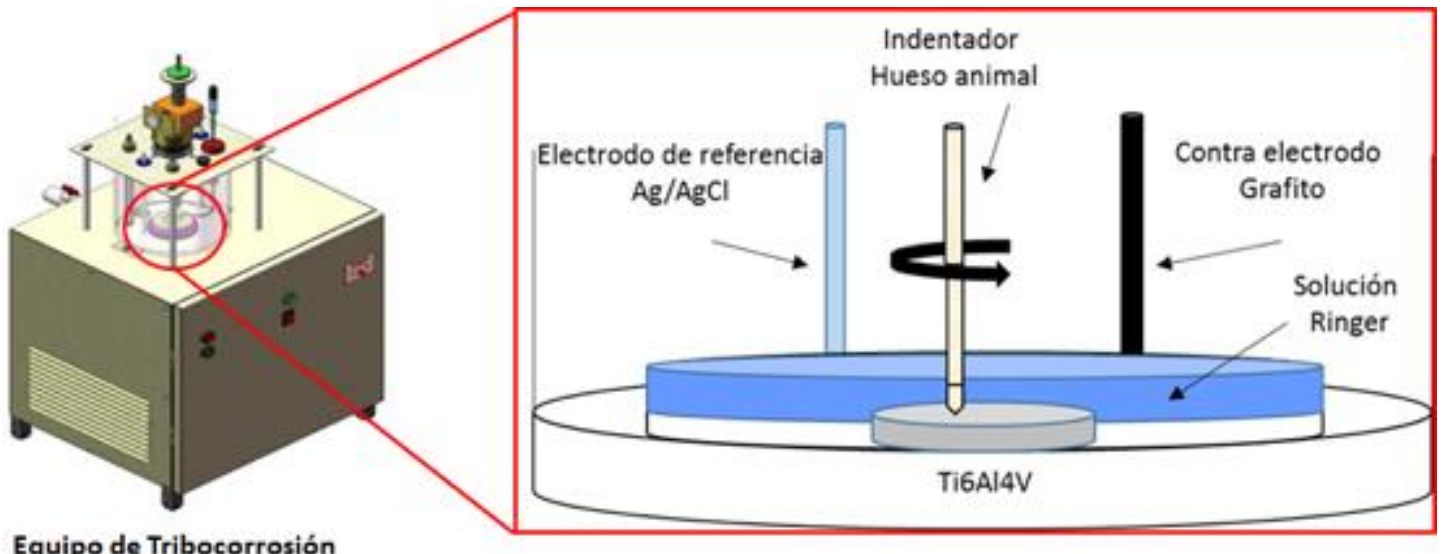

Equipo de Tribocorrosión

Figura 2: Esquema del equipo y celda empleados para los ensayos de desgaste y corrosión.

\section{RESULTADOS}

La Figura 3 presenta las curvas potenciostáticas obtenidas sobre la superficie de Ti recubierto con PLGA, estas curvas se obtuvieron manteniendo una carga constante de 0,0294 N. Como se puede observar durante el primer minuto de las pruebas las superficies muestran una disminución en la densidad de corriente, la cual luego de este corto periodo de tiempo tiene a estabilizarse en un valor aproximado de $6,5 \times 10^{-5} \mathrm{~A} / \mathrm{cm}^{2}$ en el caso de las muestras evaluadas con velocidad de rayado de $0,011 \mathrm{~m} / \mathrm{s}$ y $0,016 \mathrm{~m} / \mathrm{s}$; de la misma manera, la muestra evaluada con una velocidad de rayado de $0,022 \mathrm{~m} / \mathrm{s}$ se estabiliza en una densidad de corriente de 1,0 $\mathrm{x} 10^{-4} \mathrm{~A} / \mathrm{cm}^{2}$. Estos resultados demuestran que a mayores velocidades de rayado el recubrimiento de PLGA bajo las condiciones de estudio se degrada más rápido y no logra cumplir uno de sus propósitos el cual es de proteger el material.

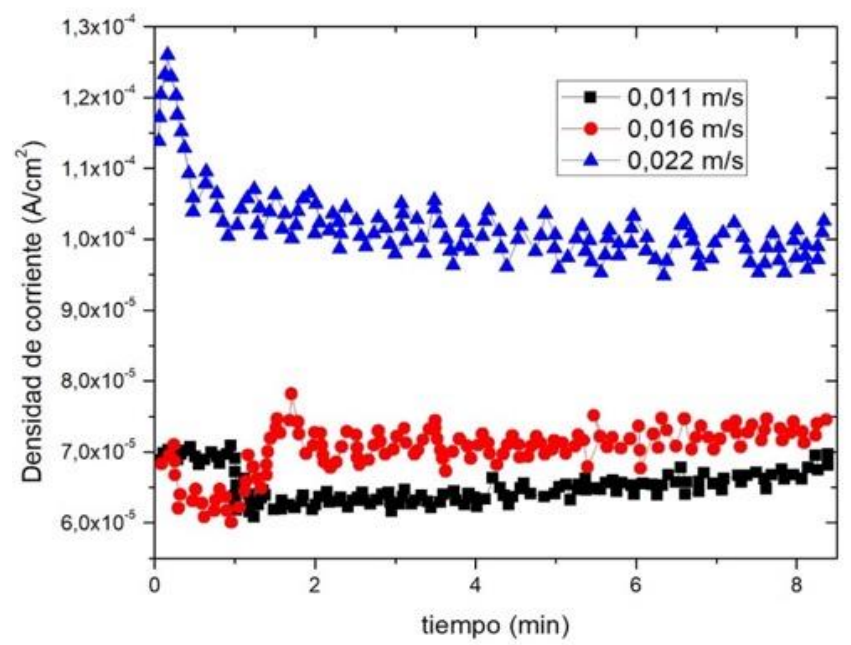

Figura 3: Curvas potenciostáticas para el Ti cp recubierto con PLGA aplicando una carga de 0,0294 N.

A continuación se presentan las curvas Tafel obtenidas para las muestras de titanio con recubrimiento PLGA (Figura 4). Como se puede apreciar, el incremento de la velocidad de giro causa un ligero desplaza- 
miento de las curvas hacia la derecha, lo cual se evidencia en un incremento de la densidad de corriente de corrosión de las muestras estudiadas. Además se puede observar que el incremento de la velocidad de giro también causa una disminución en los potenciales de corrosión lo cual se observa en el desplazamiento de las curvas hacia potenciales más negativos. Por otra parte, se puede apreciar que la carga no tiene un efecto que sea fácilmente apreciable en las curvas Tafel. Sin embargo con ayuda de los datos presentados en la Tabla 2 se puede observar que el incremento de la densidad de corriente está asociado a un incremento en la carga aplicada, donde la condición que mayor daño causa a las superficies estudiadas es la carga de 0,0294 $\mathrm{N}$ con velocidad de rayado de $0,022 \mathrm{~m} / \mathrm{s}$.

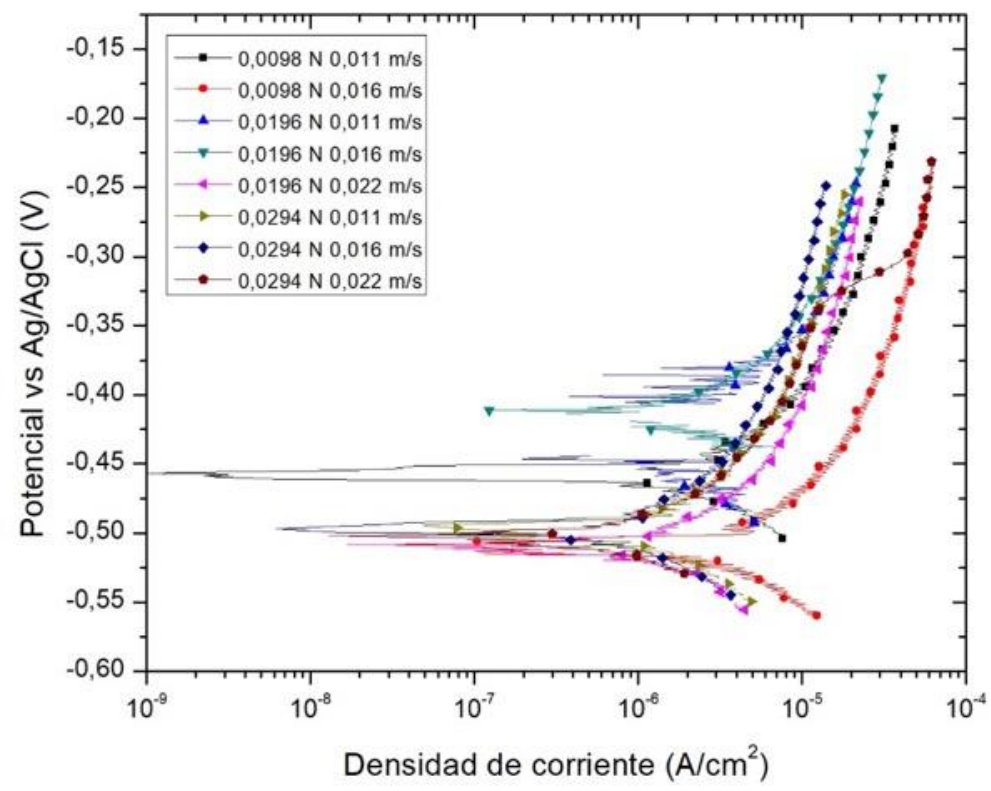

Figura 4: Curvas de Tafel obtenidas para el titanio con recubrimiento de PLGA en contacto con hueso y fluido sinovial.

Tabla 2: Parámetros electroquímicos obtenidos para los recubrimientos PLGA en contacto con hueso y fluido sinovial.

\begin{tabular}{c|c|c|c|c}
\hline Carga $(\mathbf{N})$ & Velocidad $(\mathbf{m} / \mathbf{s})$ & $\mathbf{I}_{\text {corr }}\left(\boldsymbol{\mu} \mathbf{A} / \mathbf{c m}^{\mathbf{2}}\right)$ & $\mathbf{E}_{\text {corr }}(\mathbf{m V})$ & $\mathbf{V}_{\text {corr }}(\mathbf{m p y})$ \\
\hline \multirow{3}{*}{0,0098} & 0,011 & 5,30 & $-465,5$ & 7,32 \\
\cline { 2 - 5 } & 0,016 & 5,68 & $-514,3$ & 7,84 \\
\hline \multirow{3}{*}{0,0196} & 0,011 & 5,84 & $-431,5$ & 8,06 \\
\cline { 2 - 5 } & 0,016 & 6,07 & $-420,0$ & 8,38 \\
\cline { 2 - 5 } & 0,022 & 5,83 & $-512,2$ & 8,05 \\
\hline \multirow{3}{*}{0,0294} & 0,011 & 5,85 & $-499,3$ & 8,08 \\
\cline { 2 - 5 } & 0,016 & 6,15 & $-491,2$ & 8,49 \\
\cline { 2 - 5 } & 0,022 & 6,05 & $-516,6$ & 8,35 \\
\hline
\end{tabular}

El comportamiento que se observa en las curvas Tafel, se puede asociar al desprendimiento del recubrimiento de PLGA que causa el proceso de rayado. Tanto el incremento de la carga como de la velocidad generan exposición del sustrato de titanio al electrolito lo que se ve reflejado en el incremento de los valores de densidad de corriente de corrosión. Asimismo, a mayores cargas y velocidades de rayado la capa protectora de $\mathrm{TiO}_{2}$ que el titanio forma naturalmente no logra regenerarse lo suficientemente rápido por lo que el sustrato queda expuesto y vulnerable a ser atacado por los iones corrosivos presentes en el fluido sinovial. 
En la Figura 5 se presenta la morfología y la topografía de la superficie del Ti cp recubierta con PLGA tras el ensayo de desgaste corrosión en el cual se aplicó una carga de $0,029 \mathrm{~N}$ y la velocidad de rayado fue de $0,022 \mathrm{~m} / \mathrm{s}$. Como se puede observar, el recubrimiento de PLGA fue removido de la superficie. En estas micrografías se interpretó que la parte oscura es el recubrimiento y la parte clara es la superficie descubierta. En este caso la profundidad del rayado alcanzo las $100 \mu \mathrm{m}$ desde la superficie descubierta hasta la parte superior de la capa de PLGA. El desprendimiento y la disolución del recubrimiento de PLGA en la superficie del titanio, junto a los datos electroquímicos demuestran la importancia de la velocidad de giro y la carga aplicada en la degradación del material. Esto permite atribuir el daño de la superficie de Ti cp recubierto con PLGA a un mecanismo combinado de desgaste por erosión del polímero y por abrasión por el contacto con el hueso.

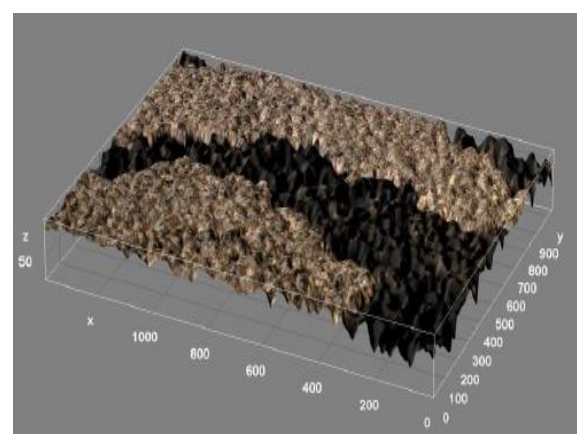

Figura 5: Morfología de la superficie de Ti cp con recubrimiento PLGA después del ensayo de desgaste corrosión $100 \mathrm{X}$.

La Figura 6 muestra las distribuciones de componentes de desgaste, corrosión y sinergismo del titanio con recubrimiento de PLGA. Los datos sugieren que la pérdida de material de una superficie metálica con PLGA que se desgasta y corroe simultáneamente excede la suma de la pérdida de material por sólo desgaste aislado y la pérdida de material por sólo corrosión. Sin embargo, esto demuestra claramente que, aparte de los mecanismos individuales resultantes del desgaste y la corrosión, la interacción sinérgica entre ambos fenómenos tiene una gran influencia en el proceso de degradación total. Esto indica que el PLGA no se opone principalmente al daño inicial de la superficie metálica, y su resistencia disminuye al contacto con la solución, por lo que la pérdida total de material se agrava por el daño sobre la superficie metálica causado por el indentador.

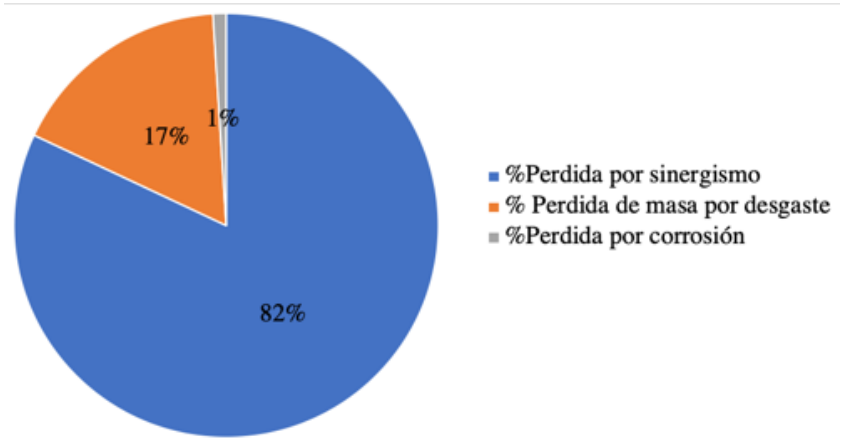

Figura 6: Diagrama de distribución de componentes de desgaste, corrosión y sinergismo.

Por último, en la Figura 7 se muestra el mapa de los regímenes de desgaste y corrosión para el titanio con re cubrimiento de PLGA. Los mapas de regímenes de desgaste-corrosión se basan en el estudio de pérdida de masa en los materiales. Con el mapa propuesto se pueden obtener las tasas de desgaste asociadas a los fenómenos que intervienen en el material, donde se observan las zonas de los regímenes en función de la carga y la velocidad aplicadas. Se puede observar cada una de las condiciones experimentales, donde se presentan los diferentes fenómenos que ocurren durante el proceso de deterioro de la superficie del electrodo, lo cual permite predecir un comportamiento del daño que sufre el material bajo las condiciones experimentales que se emplearon para este estudio. Este mapa de desgaste - corrosión se obtuvo siguiendo los lineamientos 
de la norma ASTM G119-09, 2009 [22].

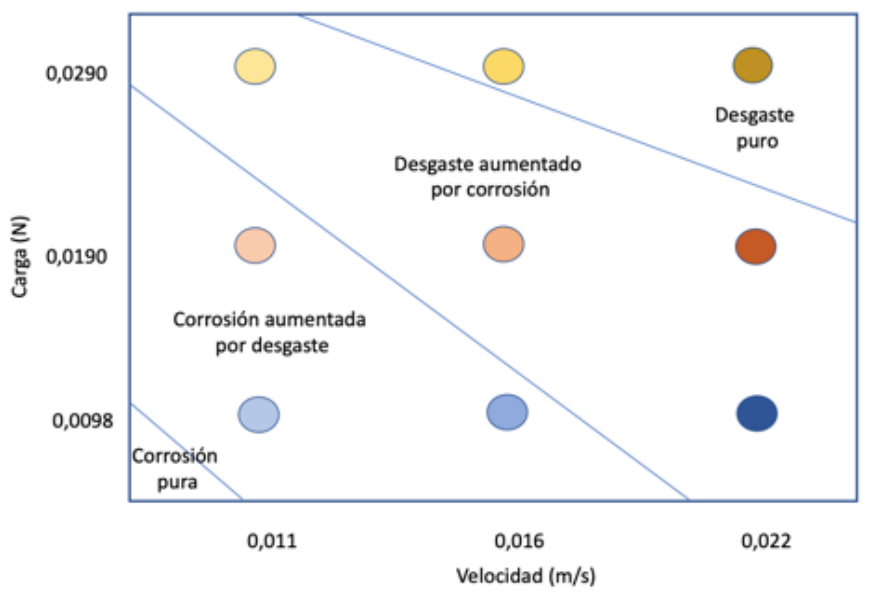

Figura 7: Mapa de regímenes de desgaste corrosión para el titanio.

Este mapa de los regímenes de desgaste-corrosión permite obtener información sobre el tipo de deterioro que tuvo la superficie del electrodo, los posibles tipos de deterioro son corrosión, corrosión aumentada por desgaste, desgaste aumentado por corrosión y desgaste puro. Para el caso del titanio con el recubrimiento de PLGA bajo las condiciones de estudio se presentó desgaste favorecido por corrosión.

\section{CONCLUSIONES}

La evaluación del sinergismo entre el desgaste y corrosión de las superficies de titanio recubierto con PLGA permitió encontrar que el sinergismo entre estos dos fenómenos para el sistema tribológico implante-huesofluido sinovial es de $82 \%$, lo cual indica que este es el proceso fundamental de la remoción de material de la superficie del electrodo.

El recubrimiento de PLGA presentó un alto índice de degradación debido al contacto con el indentador de hueso animal y a la solución Ringer, por lo cual, el mecanismo de degradación de este material es un proceso de desgaste combinado de erosión y abrasión. Sin embargo este recubrimiento mejoró la protección a la corrosión del titanio.

El mapa de desgaste - corrosión permite observar que para valores mayores de velocidad de rotación en el sistema tribológico planteado se presentaría un fenómeno de desgaste puro, al igual que para cargas mayores a las empleadas en este estudio, para los valores menores de carga y velocidad de rotación se puede presentar desgaste aumentado por corrosión.

\section{BIBLIOGRAFÍA}

[1] FAZEL M., SALIMIJAZI H. R., GOLOZAR M. A., AND GARSIVAZ JAZI M. R., “A comparison of corrosion, tribocorrosion and electrochemical impedance properties of pure Ti and Ti6Al4V alloy treated by micro-arc oxidation process," Applied Surface Science, vol. 324, pp. 751-756, 2015.

[2] GARSIVAZ JAZI M. R., GOLOZAR M. A., RAEISSI K., AND FAZEL M., "Evaluation of corrosion and tribocorrosion of plasma electrolytic oxidation treated Ti-6Al-4V alloy," Surface and Coatings Technology, vol. 244, pp. 29-36, 2014.

[3] R. AGARWAL AND A. J. GARCÍA, "Biomaterial strategies for engineering implants for enhanced osseointegration and bone repair," Advanced Drug Delivery Reviews, vol. 94, pp. 53-62, 2015.

[4] GOLDA-CEPA M., CHORYLEK A., CHYTROZS P., BRZYCHCZY WLOCH M., JAWORSKA J., KASPERCZYK J., HAKKARAINEN M., ENGVALL K., AND KOTARBA A., "Multifunctional PLGA/Parylene C Coating for Implant Materials: An Integral Approach for Biointerface Optimization," ACS Applied Materials and Interfaces, vol. 8, no. 34, pp. 22093-22105, 2016.

[5] T. KIZUKI, H. TAKADAMA, T. MATSUSHITA, T. NAKAMURA, AND T. KOKUBO, "Preparation of bioactive Ti metal surface enriched with calcium ions by chemical treatment," Acta Biomaterialia, vol. 6, no. 7, pp. 2836-2842, 2010.

[6] SMITH L. J., SWAIM J. S., YAO C., HABERSTROH K. M., NAUMAN E., AND WEBSTER T. J., 
"Increased osteoblast cell density on nanostructured PLGA-coated nanostructured titanium for orthopedic applications," International journal of nanomedicine, vol. 2, no. 3, pp. 493-499, 2007.

[7] PEÑA BALLESTEROS DARIO YESID, ESTUPIÑAN DURAN HUGO ARMANDO, CORDOBA TUTTA ELCY MARIA, MARTINEZ RICARDO, VASQUZ QUINTERO CUSTODIO, "Recubrimientos de quitosano / fosfato de calcio obtenidos por electrodeposición sobre una aleación de titanio Chitosan / calcium phosphate coating obtained by electrodeposition on titanium alloy," Revista Facultad de Ingenieria Universidad de Antioquia, vol. 54, pp. 1-9, 2010.

[8] ARDILA L. F., ESTUPIÑÁN DURAN H. A., VÁSQUEZ QUINTERO C., AND PEÑA BALLESTEROS D. Y., "Estudio De La Biodegradación Hidrolítica De Películas Delgadas De biopolimeros ceramico mediante EQCM,” En: XI Iberomet SAM Conamet 2010, pp. 1-7, Noviembre, Chile, 2010.

[9] PARADA QUINAYÁ D. C., ESTUPIÑÁN DURÁN H. A., PEÑA BALLESTEROS D. Y., VÁSQUEZ QUINTERO C., "Obtención, Electrodeposición y Caracterización de un Recubrimiento Polimérico Bioabsorbible a partir de Ácido Láctico para Aplicaciones Biomédicas," Ingeniare. Revista chilena de ingeniería, vol. $17 \mathrm{~N}^{\mathrm{o}}$ 3, pp. 365-374, 2009.

[10] GELVES JEREZ L. M., MONTALEZ SUPELANO N. D., ANAYA JAIMES Y. K., ESTUPIÑAN DURAN H. A., PEÑA BALLESTEROS D. Y., VASQUEZ QUINTERO C., "Evaluación del direccionamiento electroquímico de proteínas sobre recubrimientos de ácido poliláctico," vol. 24, no. 1, pp. 7-14, 2011.

[11] ESTUPIÑAN DURAN H. A., PEÑA BALLESTEROS D. Y., AND VASQUEZ QUINTERO C., "Osteoblast Adhesion on Scaffolds of Pla-Plg-Hydroxyapatite-Chitosan-Zinc By Electroactivation," Dyna, vol. 79, no. 176, pp. 99-104, 2012.

[12] GÓMEZ RODRÍGUEZ L. S., QUINTERO JAIME A. F., PEÑA BALLESTEROS D. Y., AND ESTUPIÑAN DURÁN H. A., "Obtención, caracterización y evaluación in vitro de recubrimientos de policaprolactona-quitosano sobre la aleación Ti6Al4V tratada químicamente, "Revista de Metalurgia, vol. 50, no. 3, p. e021, 2014.

[13] MENDES J. F., PASCHOALIN R. T., CARMONA V. B., SENA NETO A. R., MARQUES A. C. P., MARCONCINI J. M., MATTOSO L. H. C., MEDEIROS E. S., OLIVERA J. E., "Biodegradable polymer blends based on corn starch and thermoplastic chitosan processed by extrusion," Carbohydrate Polymers, vol. 137, pp. 452-458, 2016.

[14] MONTAÑEZ SUPELANO N. D., PEÑA BALLESTEROS D. Y., AND ESTUPIÑÁN DURÁN H. A., "Respuesta dieléctrica y bioquímica de un recubrimiento PLA-PGA-HAp-Quitosano-Colágeno sobre Ti6Al4V," Ingeniare. Revista chilena de ingeniería, vol. 24, no. 2, pp. 215-227, 2016.

[15] MONTAÑEZ SUPELANO N. D., GLEVEZ M, ESTUPIÑAN DURAN H. A., VASQUEZ QUINTERO C., "Evaluación de la adsorción de colágeno sobre," Revista Puentes, vol. 5, no. 2, pp. 27-32, 2011.

[16] LI J. N., CAO P., ZHANG X. N., ZHANG S. X., AND HE Y. H., "In vitro degradation and cell attachment of a PLGA coated biodegradable Mg-6Zn based alloy," Journal of Materials Science, vol. 45, no. 22, pp. 6038-6045, 2010.

[17] WANG Z., CHEN L., WANG Y., CHEN X., AND ZHANG P., "Improved Cell Adhesion and Osteogenesis of op-HA/PLGA Composite by Poly(dopamine)-Assisted Immobilization of Collagen Mimetic Peptide and Osteogenic Growth Peptide," ACS Applied Materials and Interfaces, vol. 8, no. 40, pp. 2655926569, 2016.

[18] LIU J. AND XI T., "Enhanced Anti-corrosion Ability and Biocompatibility of PLGA Coatings on MgZnYNd Alloy by BTSE-APTES Pre-treatment for Cardiovascular Stent," Journal of Materials Science and Technology, vol. 32, no. 9, pp. 845-857, 2016.

[19] CHEN G., SATO T., SAKANE M., OHGUSHI H., USHIDA T., TANAKA J., TATEISHI T., "Application of PLGA-collagen hybrid mesh for three-dimensional culture of canine anterior cruciate ligamnt cells," Materials Science and Engineering C, vol. 24, no. 6-8 SPEC. ISS., pp. 861-866, 2004.

[20] ASTM STANDAR G1-90(1999)e1, "Standard practice for preparing, cleaning, and evaluating corrosion test specimens", ASTM International, West Conshohocken, PA, DOI: 10.1520/G0001-90R99E01, http://www.astm.org.

[21] ASTM STANDAR G3-89(2010), "Standard practice for conventions applicable to electrochemical measurements in corrosion testing", ASTM International, West Conshohocke, PA, DOI:, 10.1520/G000389R10, http://www.astm.org.

[22] ASTM G119-09, "Standard guide for determining synergism between wear and corrosion", ASTM International, West Conshohocken, PA, 2009, DOI: 10.1520/G0119-09, www.astm.org. 
[23] ASTM G5-94(2011)e1, "Standard reference test method for making potentiostatic and potentiodynamic anodic polarization measurements", ASTM International, West Conshohocken, PA, 2011, DOI: 10.1520/G0005-94R11E01, www.astm.org.

\section{ORCID}

Anderson Sandoval Amador Laura Andrea Ramírez Prada Sergio Andrés Infante

Hugo Armando Estupiñán Duran Darío Yesid Peña Ballesteros
https://orcid.org/0000-0002-9929-121X

https://orcid.org/0000-0003-3932-286X

https://orcid.org/0000-0001-8782-5715

https://orcid.org/0000-0002-9607-3364

https://orcid.org/0000-0002-6193-9535 